\title{
A Systematic Review of Technology-Assisted HIV Testing Interventions
}

\author{
Keith J. Horvath ${ }^{1}$ (D) Teresa Walker ${ }^{1} \cdot$ Linda Mireles $^{1} \cdot$ Jose A. Bauermeister $^{2} \cdot$ Lisa Hightow-Weidman $^{3}$. \\ Rob Stephenson ${ }^{4}$
}

Published online: 8 June 2020

(C) Springer Science+Business Media, LLC, part of Springer Nature 2020

\begin{abstract}
Purpose of Review The purpose of this review is to describe and assess the literature on mobile health (mHealth) and other technology-based HIV testing interventions published in the 5-year period from 2015 to 2020.

Recent Findings We identified 18 published technology-based studies, 6 of which were efficacy trials and the remaining 12 were either pilot randomized controlled trials (RCTs) or quasi-experimental studies. Most $(n=10)$ interventions were conducted outside the USA, including countries in Sub-Saharan Africa $(n=4)$, China $(n=3)$, Latin America $(n=2)$, and India $(n=1)$. All efficacy trials showed some evidence of efficacy, although uptake of HIV testing was low among in intervention trials that consisted of a low number of text messages. Most pilot RCTs demonstrated high levels of feasibility and acceptability, as well as some evidence that the intervention participants benefited more than the control group. Many non-randomized trials similarly reported positive appraisal by study participants. Recommendations for future research and practice by the authors of the studies reviewed here are summarized.

Summary Technology-assisted HIV testing interventions may be an important strategy to reach national and global targets for HIV status awareness in the general population and for most at-risk groups. Although there appears to be growing evidence of their benefit, questions linger regarding how to leverage existing social media platforms to promote HIV testing, which interventions work for what populations, and best practices for scaling up mHealth and other technology-based interventions.
\end{abstract}

Keywords HIV $\cdot$ Testing $\cdot$ eHealth $\cdot$ mHealth $\cdot$ Intervention

\section{Introduction}

Globally, nearly 38 million people are estimated to be living with HIV, with 1.7 million people newly infected in 2018 and

This article is part of the Topical Collection on Behavioral-Bio-Medical Interface

Keith J. Horvath

khorvath@sdsu.edu

1 Department of Psychology, San Diego State University, San Diego, CA, USA

2 School of Nursing, University of Pennsylvania, Philadelphia, PA, USA

3 Institute for Global Health \& Infectious Diseases, University of North Carolina at Chapel Hill, Chapel Hill, NC, USA

4 Center for Sexuality and Health Disparities, and the School of Nursing, University of Michigan, Ann Arbor, MI, USA
770,000 people dying from AIDS-related illnesses in the same year [1]. Seventy-nine percent of persons globally living with HIV know their HIV-positive status, resulting in 8.1 million people who are unaware that they have HIV [1]. Central to the success of the UNAIDS 2030 95-95-95 < span > [2] targets $95 \%$ of people who are living with HIV know their status, of whom $95 \%$ receive ART, and $95 \%$ of those on ART achieve viral suppression - are innovative strategies that allow people to learn their sero-status. Conceptualized as the Ending the HIV Epidemic strategy, there is now strong programmatic and research attention focused on enabling strategies to diagnose all persons with HIV as early as possible [3] to enable rapid adoption of treatment, which will require an expansion of HIV testing services and greater utilization of those services from communities most impacted by HIV.

HIV Testing Is the First Step in the HIV Treatment and Prevention Continuum Receiving an HIV-positive diagnosis is the first step to a continuum that involves being linked to 
HIV care, prescribed antiretroviral therapy (ART), and ultimately achieve viral suppression [4]. For those that test HIVnegative, the prevention continuum includes behavioral and biomedical prevention services, including risk reduction counseling and linkage to pre-exposure prophylaxis (PrEP). In a recent study examining trends in HIV testing among US adults, $46 \%$ of adults had tested for HIV in their lifetime, with highest rates of being ever tested among Black adults ( $69 \%)$ and Latinx $(\sim 48 \%)$ persons [5]. Among those that report an HIV risk factor (5.1\% of persons; risk factors including injection drug use, a sexually transmitted infection in the past year, giving or receiving money or drugs in exchange for sex in the past year, or condomless anal sex), approximately $65 \%$ had ever tested for HIV. In the same study, $14.8 \%$ of US adults had tested for HIV in the past year, with highest rates among Black (34.1\%) and Latinx (17.6\%) adults, and persons reporting at least one risk behavior (34.2\%). A recent report estimated that $16 \%$ of US men who have sex with men (MSM) - the risk group experiencing the highest prevalence of HIV in the USA - living with HIV are unaware of their status [6]. These findings clearly demonstrate that there is substantial room for improvement to reach the CDC recommended testing guidelines of having all adults test for HIV at least once in their lifetime, and that persons (including MSM) at high risk for HIV be tested at least annually. [7]

Globally, many countries have experienced sub-optimal HIV testing rates as seen in the USA, and reaching the 9595-95 targets by 2030 [8] will not be possible without increased HIV testing efforts overall and in key populations. Approximately $79 \%$ of people living with HIV globally knew their HIV status in 2018 [9], leaving significant room for improvement in the uptake of HIV testing. In a systematic review and meta-analysis of HIV testing among MSM in Africa, an estimated $61 \%$ of MSM ever tested for HIV, $46 \%$ tested for HIV in the past 12 months, and only $18.2 \%$ of MSM living with HIV were aware of their status [10]. There was substantial variation by region, such that MSM in East Africa, which has laws criminalizing same-sex relations, had the lowest levels of past year HIV testing (37.1\%) and HIV-positive awareness $(10.1 \%)$ [10]. In China, the estimated percentage of the population who are aware of their HIV status is approximately $54 \%$, with a slightly higher percentage of MSM (59\%) and sex workers (56\%) knowing their status [11]. Factors associated with greater HIV testing in China include higher educational level, participation in HIV education programs, and sexual orientation disclosure [12]. A recent review also concluded that the scale up of HIV testing programs, including HIV self-testing (HIVST), is critical to address the HIV epidemic in Latin America [13].

The use of technology to deliver HIV prevention and treatment interventions is now common due to the widespread integration of technology in daily life, both in the USA and globally $[14,15]$. Technology-delivered interventions have the ability to reach a broad audience, facilitate rapid scalability, promote consistent and "real-time" delivery, have relatively low implementation costs, and overcome the limitations of face-to-face interventions [16]. In addition to these benefits, HIV self-testing (HIVST) kits may reduce physical and psychosocial barriers to testing, including stigma and confidentiality concerns [17]. A review of studies (published from January 2016 through May 2017) in which technology was used to increase or improve the experience of HIV self-testing (HIVST) among MSM showed that technology was used in several ways: HIVST kits were distributed through existing social media platforms (e.g., Grindr), the distribution of HIVST kits through comprehensive technology-based HIV interventions (mobile applications or "apps"), and the use of technology platforms (e.g., video counseling) to support the correct use of HIVST kits [18]. While informative, this review was limited in scope (i.e., technology-assisted HIVST programs) and because it was limited to the past year and a half of research. A broader review of published technologyassisted HIV testing interventions would provide a more comprehensive perspective on the current status of the field.

The purpose of this review is to describe and assess the literature on mobile health (mHealth) and other technologybased HIV testing interventions published in the 5-year period from 2015 to 2020 . The review is intended to promote understanding of existing efforts that leverage technology to address HIV testing, as well as provide potential direction for future research and practice in this emerging area.

\section{Search Strategy}

We searched PubMed for publications using the search strategy outlined in Fig. 1. In our initial search, 178 articles were displayed using the available filters (e.g., human subjects; within the past 5 years) offered by PubMed. The abstracts for these studies were reviewed by two authors, which narrowed the list of eligible studies to 45 . The full article of those 45 publications was retrieved and reviewed for inclusion in this review. Articles that provided findings of interventions that either completely or partially used technology (e.g., text messaging, online websites, mobile applications; social media platforms) to facilitate intervention delivery were included in this review; studies that exclusively used discussions by telephone as their delivery format were excluded from consideration. Moreover, articles were excluded if they were pre-intervention formative studies, study protocols, review articles, or secondary analysis articles. This process yielded 11 articles from the PubMed search that met inclusion criteria. An additional seven articles were identified through a review of the reference lists and expert suggestion for inclusion in this review, for a total of 18 included studies (Tables 1 and 2). The discussion below is organized by intervention design, beginning with more rigorous randomized 
controlled trials (RCTs) to assess intervention efficacy. Next we describe the results from pilot RCTs, and finish with quasiexperimental and qualitative studies. A brief review of what recommendations for research and practice are made by the authors of those studies is reviewed here to provide possible insights into future directions for research and practice.

\section{HIV Testing Intervention Efficacy Trials}

Six articles reported the results of efficacy of randomized controlled trials (Table 1), with sample sizes ranging from 430 [19••] participants to 2262 [20] participants. Four of the six interventions were conducted with MSM, one of which was in the USA [21]. The only efficacy trial of an HIV testing intervention among US MSM, CyBER/testing, used live chat discussion through sites where MSM meet others for sex and relationships (e.g., Adam4Adam) to discuss HIV prevention and testing options [21]. Other interventions for MSM outside of the USA were conducted in China [22••] and Peru [19••].

Overall, three-quarters of interventions provided HIVST as a testing option, and all were conducted in non-US settings (China, Kenya, Peru, Tanzania). Some intervention trials leveraged social media platforms to deliver HIVST messaging and kits. For example, Tang [22・•] used WeChat, a popular messaging platform in China, to deliver information and promotional materials about HIVST to MSM. Similarly, Young et al. [23] identified peer mentors to deliver HIV prevention messages to adult MSM on Facebook over the course of 12 weeks. Wang [19••] mailed HIVST kits to MSM, and provided participants online counseling in which participants engaged in pre-test counseling, supervised HIV self-testing, and post-test counseling. Two trials focused on participants who were not MSM and also provided the option of using HIVST kits among high-risk groups in Sub-Saharan Africa. For example, Kelvin et al. [20] provided male truckers with three text messages spaced a week apart to promote the availability of HIVST kits. This approach was also used among female sex workers [24].

Fig. 1 PubMed search strategy
All studies showed some evidence of efficacy (i.e., the intervention successfully modified HIV testing behaviors). For example, Tang and colleagues crowdsourced HIV testing content for MSM and distributed those messages biweekly using WeChat in eight cities in China. Compared with historical HIV testing rates in those same cities, intervention participants who received messages about HIV testing over WeChat were found to have increased their HIV testing by $8.9 \%$ within

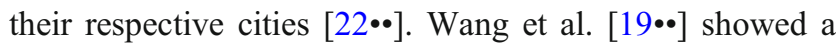
nearly $40 \%$ difference in any HIV testing at month 6 between intervention and control conditions in their online HIV counseling and testing intervention. The intervention also outperformed the control in both interventions that used an existing commercial social media platform for intervention delivery $[21,23]$. The intervention approach used by Kelvin and his colleagues in Sub-Saharan Africa [20,24]-namely, text messaging to promote in-clinic and at-home HIVSTshowed statistically significant differences in favor of the intervention condition; however, testing rates were extremely low (1.8\% for male truckers and $7.5 \%$ of female sex workers) which calls into doubt the feasibility of this approach.

\section{HIV Testing Pilot Designs}

Twelve intervention studies were included as either pilot trials or used quasi-experimental designs (Table 2). Of those, half $(n=6)$ were pilot RCTs [25-27, 28・, 29, 30], with sample sizes between 100 [26] and 302 [27]. Most $(n=5)$ pilot RCTs were conducted in the USA [25, 27, 28•, 29, 30], with one other conducted in China [26]. Likewise, five of the six pilot RCTs were with MSM [25-27, 29, 30]. Intervention delivery of the pilot RCTs varied in format: webapps (i.e., websites that are mobile optimized) [25, 30], native mobile apps [26, 29], online counseling [28•], and text messaging [27]. Interventions tested through a pilot RCT study design also differed in their types of content and features. For example, Bauermeister [25] showed young (ages 15-24) MSM a series of sequential webpages, in which participants were

Search Strategy: Article must be published in English within the last 5 years, report the primary results of an HIV intervention study, report the results of the HIV testing intervention either quantitatively or qualitatively, include human subjects, include "intervention" and test" in title or abstract, and include $\geq 1$ term from each group.

Group 1: Non-mesh: "online," "technology," "mhealth" Mesh: "social networking," "telecommunications," "computer communication networks"

Group 2: Non-mesh: "HIV prevention," "HIV testing" Mesh: "HIV Infections/prevention and control," "HIV Infections," "HIV"

Pubmed search details: (("online" OR "technology" OR "social networking"[mesh] OR "mhealth" OR "telecommunications" [mesh] OR "Computer Communication Networks"[Mesh]) AND ("HIV Infections/prevention and control"[Majr] OR HIV Infections[MAJR] OR HIV[MAJR] OR "HIV prevention" OR "HIV testing")) AND intervention[Title/Abstract] AND test*[Title/Abstract] 


\begin{tabular}{|c|c|c|c|c|}
\hline 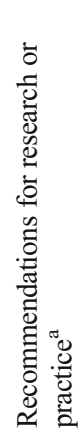 & 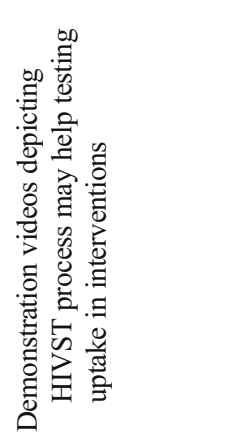 & 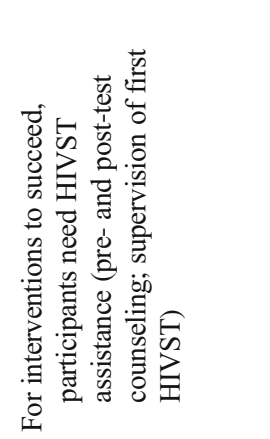 & 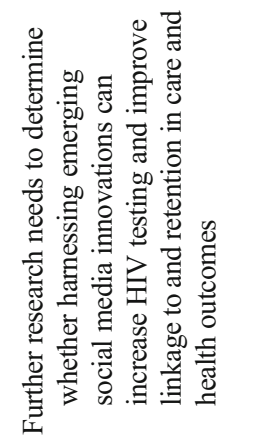 & 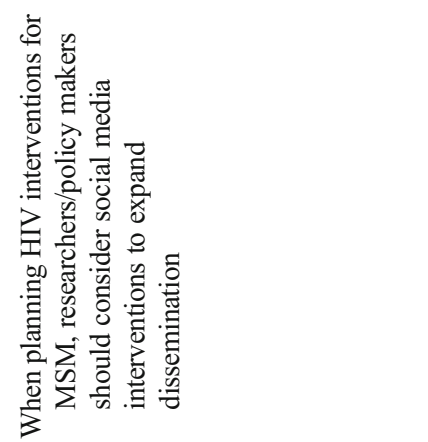 \\
\hline$\frac{n}{z}$ & 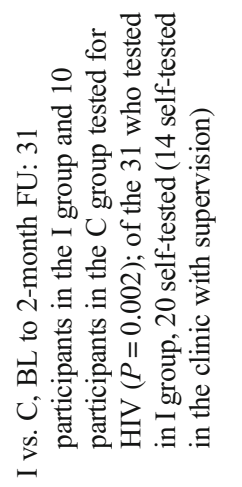 & 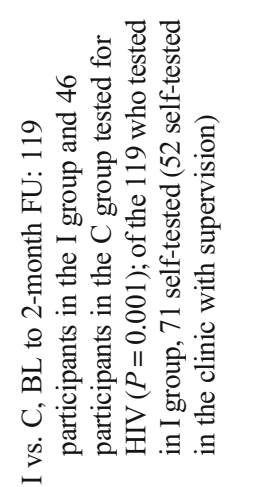 & 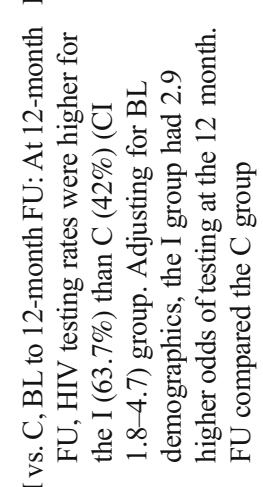 & 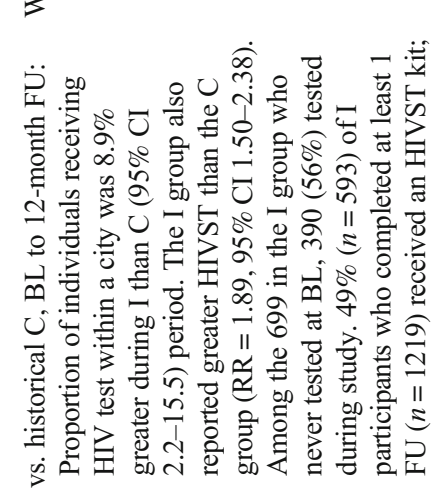 \\
\hline 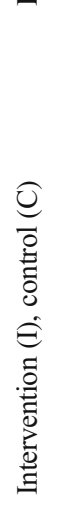 & 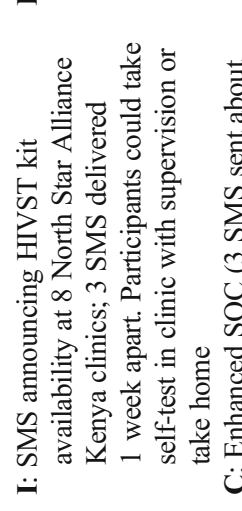 & 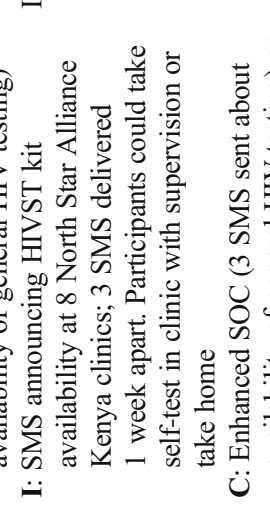 & 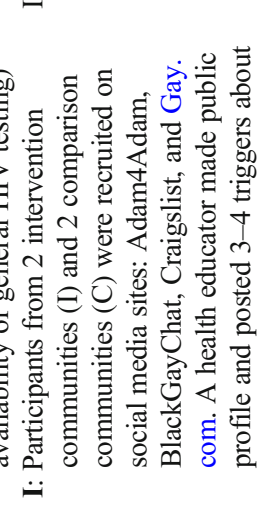 & 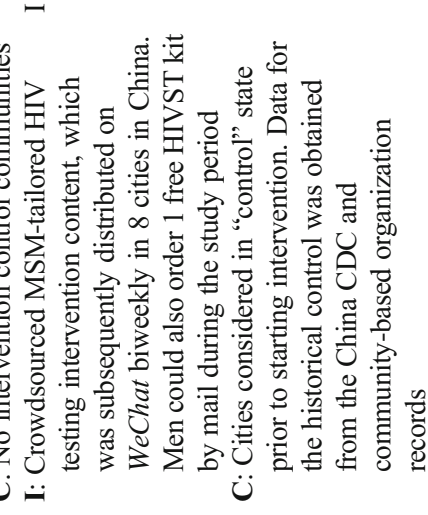 \\
\hline 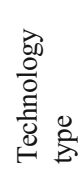 & 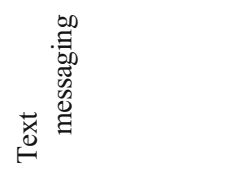 & 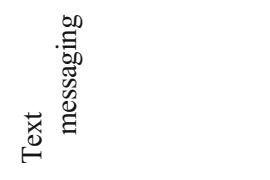 & 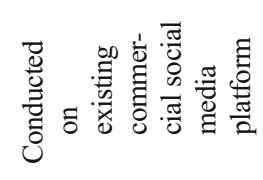 & 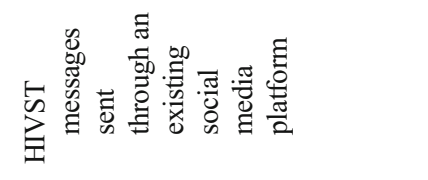 \\
\hline 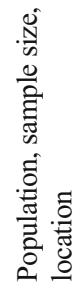 & 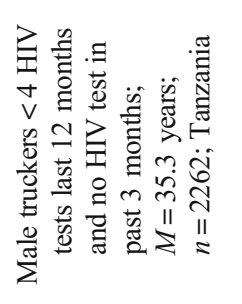 & 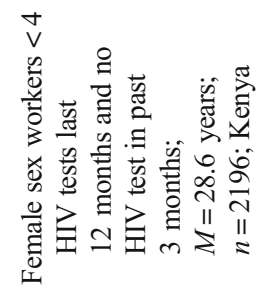 & 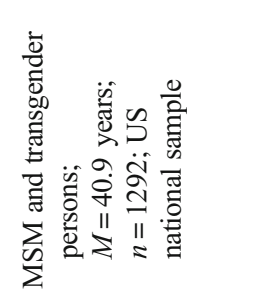 & 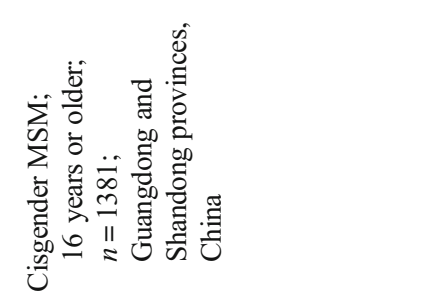 \\
\hline 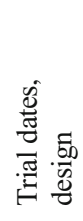 & 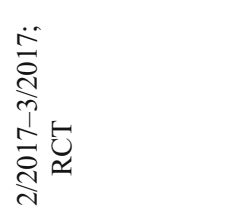 & 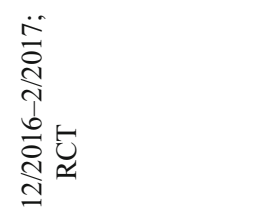 & 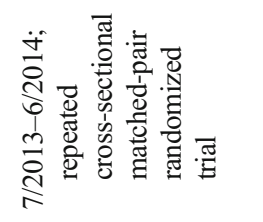 & 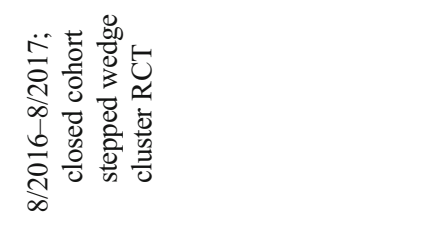 \\
\hline 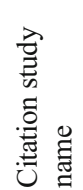 & 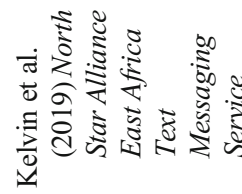 & 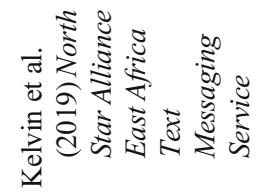 & 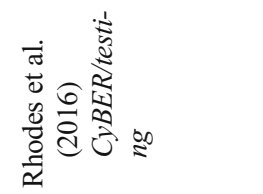 & 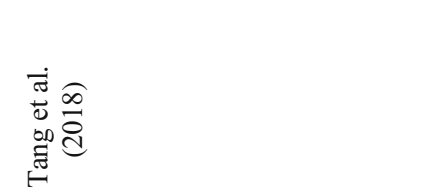 \\
\hline
\end{tabular}




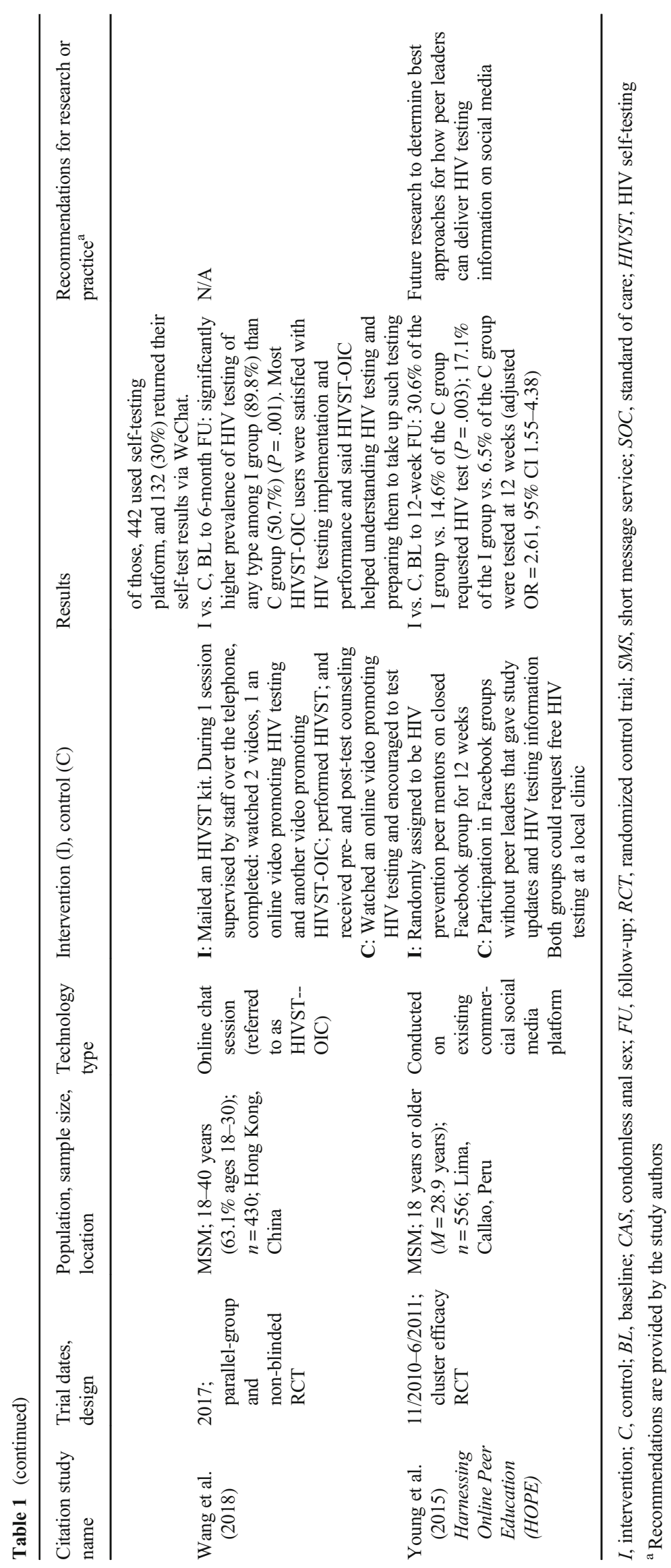




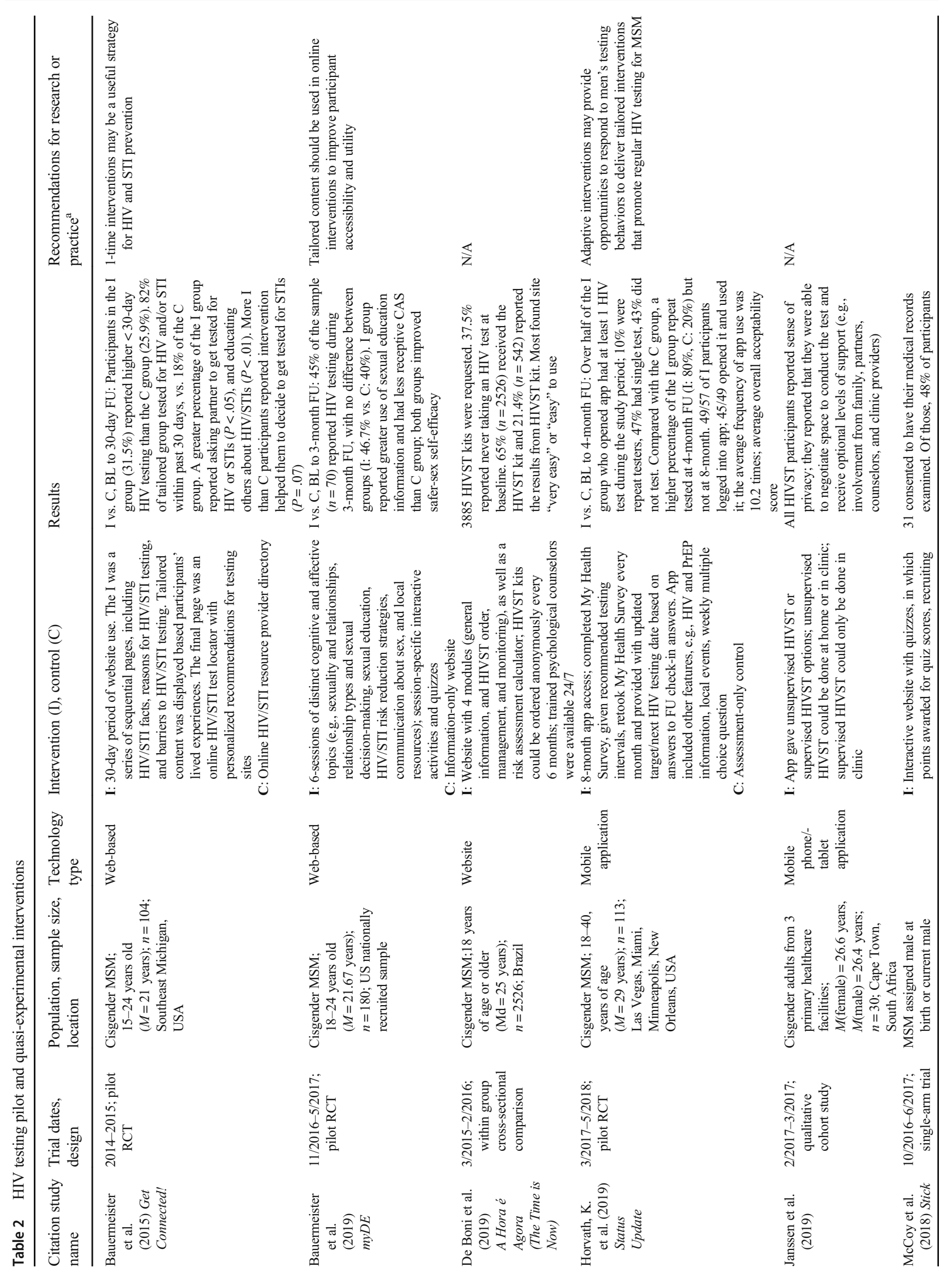




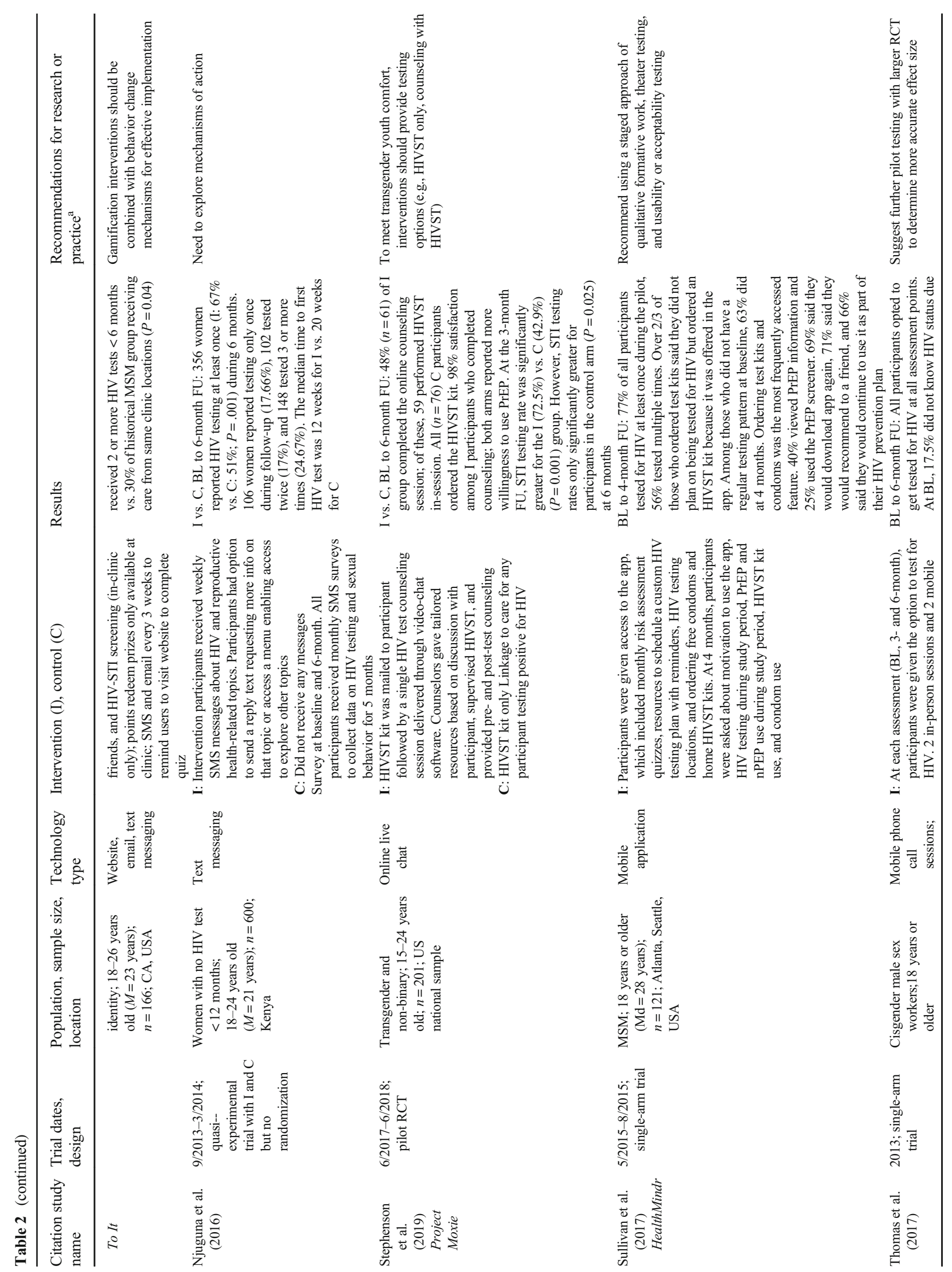




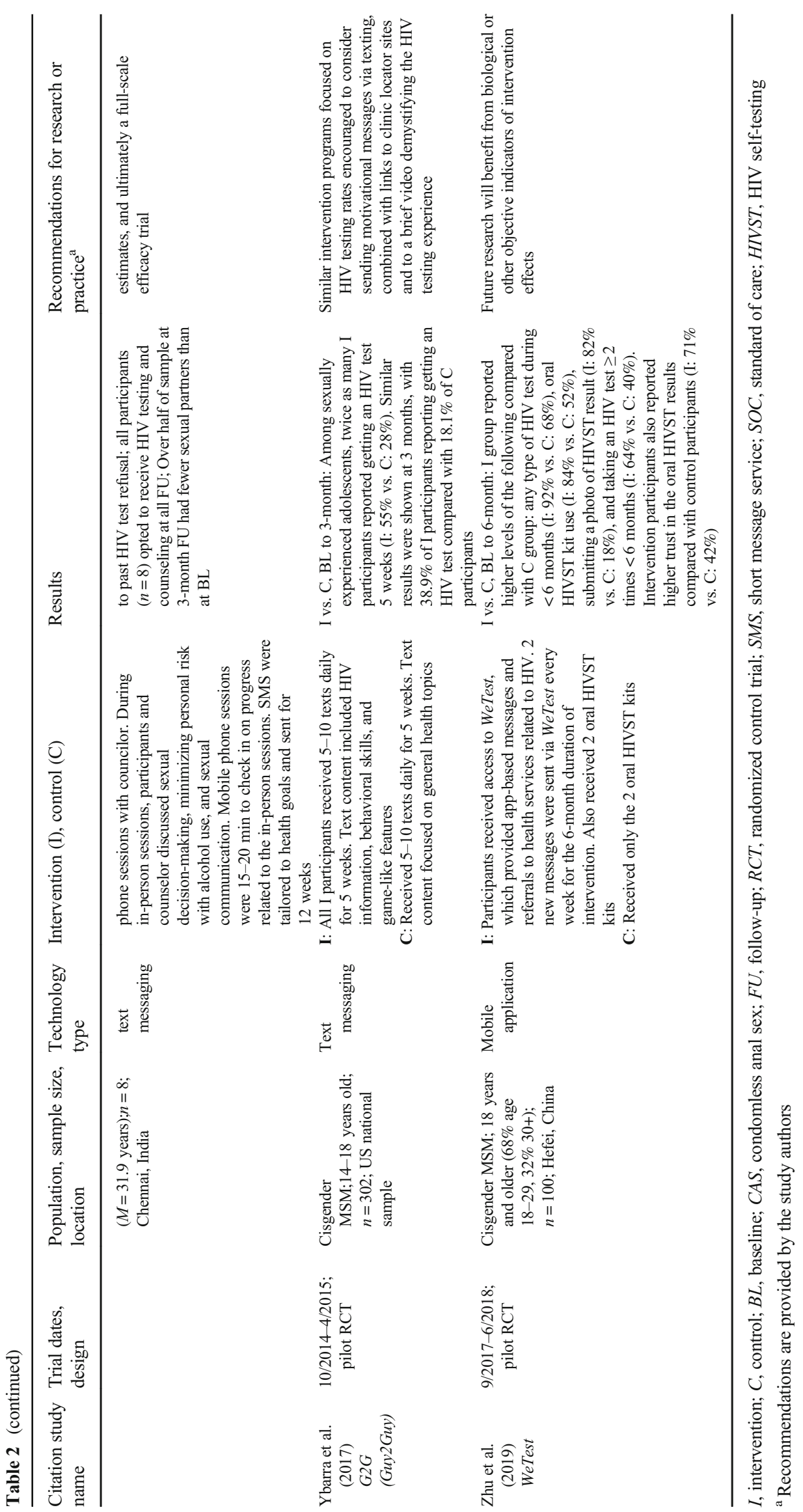


presented with tailored content depending on their life circumstances and attitudes. The final page of the intervention was a list of HIV/STI clinics and providers that is personalized to the youth's preferences. In contrast, Stephenson and his colleagues [28.] used a single-session online counseling session with transgender youth to perform supervised HIVST, preand post-test counseling, and problem solve barriers to engage in HIV prevention. In the Guy2Guy intervention [27], adolescent MSM were sent 5-10 theoretically grounded text messages per day for five weeks. Other intervention activities used in pilot RCTs included a personalized HIV testing date recommender [29], HIV testing messages and referrals to HIV health services [26], and the provision of sexual health and relationship information [30].

Several $(n=4)$ pilot RCTs showed some evidence that the intervention had a positive effect on testing behaviors. For example, in the Bauermeister [25] study described above, a higher percentage of young MSM in the intervention group tested at the 30-day follow-up assessment than those in the control (32\% vs. $26 \%$ ). Other studies showed higher rates of repeat HIV testing at four months for adult MSM [29], any type of testing among adolescents at five weeks [26, 27], and HIVST kit use at six months [26]. Other pilot RCTs found either no difference in HIV testing between groups [30] or had a relatively low uptake of the HIVST online counseling intervention by intervention participants [28•]. Of the four studies that collected participant ratings of the acceptability and satisfaction with the intervention, acceptability ratings were average to high $[25,28 \bullet, 29,30]$. Interestingly, the only HIV testing intervention tailored for transgender and non-binary youth showed sub-optimal levels of intervention uptake (48\% of those randomized to the intervention group attended the online HIVST counseling session); however, there was high satisfaction among youth who completed the online counseling session $\left[28^{\bullet}\right]$.

\section{HIV Testing Single-Arm, Quasi-Experimental, and Qualitive Designs}

Non-RCT intervention approaches $(n=6)$ included singlearm trials [31, 32•, 33], cross-sectional comparison studies [34•], qualitative studies of intervention acceptability [35], and a two-arm study without randomization [36•] (Table 2). Unlike the pilot RCTs, the majority of non-randomized studies $(n=4)$ were conducted in settings outside the USA, including Brazil [34•], South Africa [35], Kenya [36•], and India [31]. However, similar to pilot RCTs, most $(n=4)$ nonrandomized studies were tailored for MSM [31, 32•, 33, $34 \bullet]$. Intervention delivery among these studies was similarly diverse as that of the pilot RCT, and included websites [34•], mobile apps [32•,35], text messaging [36•], and a combination of various technologies [31, 33]. De Boni et al. [34•] created a website where adult MSM received general HIV and health information across several modules and could order and receive information about HIVST. A mobile app (HealthMindr) was used by Sullivan and his colleagues to allow adult MSM to assess their risk for HIV, get PrEP information, create HIV testing plans, and order free condoms and HIVST kits [32•]. In contrast, a variety of technologies were used in the single-arm trial of an intervention in Chennai, India, for male sex workers; those included mobile phone sessions and the use of text messaging, in addition to inperson meetings [31].

The non-RCT intervention approaches generally showed high uptake of HIV testing and HIVST. For example, among over 2500 MSM in Brazil visiting an HIVST website (38\% of whom had never been tested for HIV), three-quarters requested an HIVST kit, although only a minority reported the results of their HIVST [34•]. Among 600 adult women in Kenya who were non-randomly assigned to receive weekly text messages and reply for more information or to not receive such messages, a higher proportion of women receiving the messages reported getting tested at least once during the next six months than those who did not (67\% vs. 51\%) [36•]. Even higher rates of testing (77\%) were shown among 121 US-based MSM using the HealthMindr mobile app across a four-month period [32•]. Most studies reported participant's level of acceptability and satisfaction $[31,32 \cdot 33,34 \bullet, 35]$ with the intervention. Satisfaction and acceptability levels were overall moderate to high, noting across studies the easy-to-use features, privacy that the interventions provide, and their willingness to recommend the intervention.

\section{Recommendations for Practice and Research}

Recommendations for research and practice provided by the authors of the studies included in this review are provided in the final column of Tables 1 and 2. Recommendations for research and practice includes specific intervention components to promote HIV testing, including tailoring [30], gamification strategies [33], and the use of motivational text messages [27]. With respect to intervention platform and design, the potential for the use of existing social media platforms to deliver intervention content was noted [21, 22••, 23]. Similarly, study designs that were recommended for further study and possible use include brief interventions [25], adaptive designs [29], and those that provide a number of different technology-enabled options from which users could choose [28•]. Relatedly, one study [32•] called for a staged approach to intervention development that includes qualitative work, theater testing, usability testing, and pilot and efficacy testing. A number of studies noted the need for innovations that helped participants successfully navigate the HIV and HIVST process, including the possible use of demonstration videos $[20,27]$ and supervision of the testing process the first time to ensure correct use [24]. Finally, others expressed a 
need to understand intervention mechanisms of action [36 ] and for objective biomarkers of intervention effects [26].

\section{Conclusion}

HIV testing is a foundational first step in the entry into the HIV prevention and treatment continuum. This review found 18 published technology-assisted intervention studies that support the uptake of testing, enhance the experience and correct use of HIV testing, and directly assist with the delivery of HIVST kits. Similar to other reviews of mHealth and other technology-based interventions for HIV prevention or treatment outcomes [37,38], this review of studies in the past five years showed that most reported the results of pilot or quasiexperimental study designs $(n=12)$. Of the efficacy RCT trials, the majority were conducted in non-US settings including Sub-Saharan Africa, China, and Peru, and those that were tailored to MSM showed the strongest evidence in favor of the intervention. The two studies conducted in Tanzania and Kenya used a similar intervention delivery method, which consisted of sending a relatively small number of text messages (three) to encourage HIVST kit use either at a clinic with supervision by a healthcare worker or alone [20,24]. This approached failed to engage most participants, suggesting the need for more intensive strategies to promote HIV testing in Sub-Saharan Africa.

Among these efficacy trials, the use of an existing social media platform (e.g., Grindr or Facebook) to engage high-risk MSM and encourage HIV testing was supported. Rhodes et al. [21], Tang et al. [22••], and Young et al. showed positive intervention effects using social media platforms to identify and enroll MSM into their studies. The use of existing commercial social media platforms to advertise and recruit MSM into HIV prevention and treatment studies is common [39]; however, how to leverage these platforms as part of intervention delivery is more complex. On the one hand, two of the studies reviewed here show strong support for engaging highrisk MSM through existing social media platforms to encourage them to test offline and order HIVST kits. On the other hand, maintaining a high level of privacy with respect to HIV status and MSM identity is an important consideration in HIV testing interventions [40,41], and it is not yet clear whether changing platform guidelines and users' beliefs about privacy on social media platforms will impede their use for HIV testing. This review found intervention studies that used other delivery technologies - such as mobile apps [32•], text messaging [27], or websites [42] — to encourage HIV testing and distribute HIVST kits. While text messages can be automated to participants on a chosen schedule, mobile apps and websites require users to actively engage (e.g., open the app or search the web) with the technology, which can be a challenge. The choice of a technology-based intervention delivery modality (or modalities) is, in part, driven by the capacity to access that technology in a given region and the adoption of that technology in communities. In the context of studies reviewed here, text messaging was the single modality used in studies conducted in Sub-Saharan Africa, where mobile phone adoption is high but where capacity to access the internet via mobile devices and use mobile applications is still not widespread [43]. In contrast, US-based studies included the use of diverse technology-based modalities, possibly reflecting both high capacity to enable these technologies and high adoption communities who would benefit from HIV testing [44]. Given the relatively small number of studies overall and the small number of studies conducted in non-US settings, it is difficult to make specific recommendations for which modalities will ultimately prove to be most impactful. Therefore, continued efforts are needed to determine which technology-based modalities and intervention components will sustainably enable HIV testing across diverse communities.

Across studies, response to testing interventions was not uniform. Studies that used technology for participants to order and/or receive HIVST kits tended to have higher rates of testing $[26,32 \cdot]$ than interventions that encouraged HIV testing (presumably at clinics or other offline venues) without the provision of an HIVST kit [29, 30, 36 $]$. As noted by several authors of the studies reviewed here [28•,29], there is a need for study designs and community programs to offer a variety of testing interventions that can support persons who need minimal support to test and also identifying and providing timely interventions to those who require more intensive supports to do so. In a research setting, this may be done through sequential multiple assignment randomization trial (SMART) designs [45], while in community settings this may be accomplished by providing a menu of testing services (e.g., regular text messages for regular testers and technology-assisted peer navigation for hard-to-engage persons) depending on the client's testing history and motivation.

We did not identify any studies that were implementation (i.e., "real-world") trials of HIV testing interventions, perhaps reflecting the nascent stage of the science. Ultimately, to impact rates of HIV testing to meet the Ending the HIV Epidemic and to achieve the 95-95-95 goals [3], we need a much greater understanding of how the approaches reviewed here can be integrated into communities. This seems especially true for rural areas where HIV burden is often very high [46, 47]. One of the many promises of technology-based interventions is the ability to reach persons who are geographically diverse and isolated [16]. Thus far, however, there is little empirical evidence for how to scale up such efforts, which should be a primary focus of investigation to advance the field of HIV testing and to make a measurable impact on rates HIV testing. These efforts are particularly critical given the immediate and long-term impacts of the COVID-19 pandemic, which has 
both forced researchers to quickly adapt to online recruitment, enrollment, and intervention delivery strategies [48] and to consider the continuing needs of high-risk communities who will require remotely delivered service [49]. As the entrance into the HIV prevention and treatment continuum, maximizing testing behaviors among at-risk groups should have positive downstream impacts on other steps in the continuum. Our review suggests that technology should be leveraged to achieve these HIV continuum goals globally.

Funding Information K.J. Horvath is supported by the National Institute of Mental Health (R34MH1 16878). J.A. Bauermeister is supported by the Penn Center for AIDS Research (CFAR), an NIH-funded program (P30 AI 045008). K.J. Horvath, J.A. Bauermeister, L. Hightow-Weidman, and R. Stephenson are funded by the National Institute of Child and Human Development (U19HD089881)

Data Availability All studies reviewed here are publicly available.

\section{Compliance with Ethical Standards}

Conflict of Interest The authors declare that they have no conflicts of interest.

\section{References}

Papers of particular interest, published recently, have been highlighted as:

- Of importance

•. Of major importance

1. UNAIDS. Global HIV \& AIDS statistics - 2019 fact sheet. 2019 [cited 2020 February 4]; Available from: https://www.unaids.org/ en/resources/fact-sheet.

2. UNAIDS. Fast track: ending the AIDS epidemic by 2030. 2014; Available from: https://www.unaids.org/sites/default/files/media asset/20140925_Fast_Track_Brochure.pdf. [Accessed 3 June 2020]

3. Fauci AS, Redfield RR, Sigounas G, Weahkee MD, Giroir BP. Ending the HIV epidemic: a plan for the United States. JAMA. 2019;321(9):844-5.

4. Centers for Disease Control and Prevention. HIV care saves lives: vital suppression is key. 2014 [cited 2019 June 5]; Available from: https://www.cdc.gov/vitalsigns/hiv-aids-medical-care/index.html.

5. Patel D, Johnson CH, Krueger A, Maciak B, Belcher L, Harris N, et al. Trends in HIV testing among US adults, aged 18-64 years, 2011-2017. AIDS Behav. 2020;24(2):532-9.

6. Harris NS, Johnson AS, Huang YLA, Kern D, Fulton P, Smith DK, et al. Vital signs: status of human immunodeficiency virus testing, viral suppression, and HIV preexposure prophylaxis - United States, 2013-2018. MMWR Morb Mortal Wkly Rep. 2019;68(48):1117-23.

7. Branson B, et al. Revised recommendations for HIV testing of adults, adolescents, and pregnant women in health-care settings. MMWR Recomm Rep. 2006;55(RR-14):1-17 quiz CE1-4.

8. UNAIDS. Fast track: ending the AIDS epidemic by 2030. 2014 [cited 2020 February 4]; Available from: https://www.unaids.org/
sites/default/files/media_asset/20140925_Fast_Track_Brochure. pdf.

9. HIV.gov. The global HIV/AIDS epidemic. 2019 [cited 2020 February 10]; Available from: https://www.hiv.gov/hiv-basics/ overview/data-and-trends/global-statistics.

10. Stannah J, Dale E, Elmes J, Staunton R, Beyrer C, Mitchell KM, et al. HIV testing and engagement with the HIV treatment cascade among men who have sex with men in Africa: a systematic review and meta-analysis. Lancet HIV. 2019;6(11):e769-87.

11. UNAIDS. China. 2020 [cited 2020 February 10]; Available from: https://www.unaids.org/en/regionscountries/countries/china.

12. Ritchwood TD, He J, Smith MK, Tang W, Ong JJ, Oduro A, et al. "Getting to Zero" among men who have sex with men in China: a review of the HIV care continuum. Curr HIV/AIDS Rep. 2019;16(6):431-8.

13. Luz PM, Veloso VG, Grinsztejn B. The HIV epidemic in Latin America: accomplishments and challenges on treatment and prevention. Curr Opin HIV AIDS. 2019;14(5):366-73.

14. Cao B, Gupta S, Wang J, Hightow-Weidman LB, Muessig KE, Tang W, et al. Social media interventions to promote HIV testing, linkage, adherence, and retention: systematic review and meta-analysis. J Med Internet Res. 2017;19(11):e394.

15. Knight R, et al. Online interventions to address HIV and other sexually transmitted and blood-borne infections among young gay, bisexual and other men who have sex with men: a systematic review. J Int AIDS Soc. 2017;20(3):e25017.

16. Simoni JM, Kutner BA, Horvath KJ. Opportunities and challenges of digital technology for HIV treatment and prevention. Curr HIV/ AIDS Rep. 2015;12(4):1-4.

17. Qin Y, Han L, Babbitt A, Walker JS, Liu F, Thirumurthy H, et al. Experiences using and organizing HIV self-testing. Aids. 2018;32(3):371-81.

18. LeGrand S, Muessig KE, Horvath KJ, Rosengren AL, HightowWeidman LB. Using technology to support HIV self-testing among MSM. Curr Opin HIV AIDS. 2017;12(5):425-31.

19.• Wang $Z$, et al. A randomized controlled trial evaluating efficacy of promoting a home-based HIV self-testing with online counseling on increasing HIV testing among men who have sex with men. AIDS Behav. 2018;22(1):190-201 This efficacy RCT demonstrated that online video counseling sessions may be an important way to reach and test at-risk MSM in China.

20. Kelvin EA, George G, Kinyanjui S, Mwai E, Romo ML, Oruko F, et al. Announcing the availability of oral HIV self-test kits via text message to increase HIV testing among hard-to-reach truckers in Kenya: a randomized controlled trial. BMC Public Health. 2019;19(1):7.

21. Rhodes SD, McCoy TP, Tanner AE, Stowers J, Bachmann LH, Nguyen AL, et al. Using social media to increase HIV testing among gay and bisexual men, other men who have sex with men, and transgender persons: outcomes from a randomized community trial. Clin Infect Dis. 2016;62(11):1450-3.

22.• Tang W, et al. Crowdsourcing to expand HIV testing among men who have sex with men in China: a closed cohort stepped wedge cluster randomized controlled trial. PLoS Med. 2018;15(8): e1002645 This efficacy RCT study demonstrated that social media may be an important means to deliver HIV testing messages and provide HIVST kits to at-risk MSM in China.

23. Young SD, Cumberland WG, Nianogo R, Menacho LA, Galea JT, Coates T. The HOPE social media intervention for global HIV prevention in Peru: a cluster randomised controlled trial. Lancet HIV. 2015;2(1):e27-32.

24. Kelvin EA, George G, Mwai E, Kinyanjui S, Romo ML, Odhiambo $\mathrm{JO}$, et al. A randomized controlled trial to increase HIV testing demand among female sex workers in Kenya through announcing the availability of HIV self-testing via text message. AIDS Behav. 2019;23(1):116-25. 
25. Bauermeister JA, Pingel ES, Jadwin-Cakmak L, Harper GW, Horvath K, Weiss G, et al. Acceptability and preliminary efficacy of a tailored online HIV/STI testing intervention for young men who have sex with men: the Get Connected! program. AIDS Behav. 2015;19(10):1860-74.

26. Zhu X, Zhang W, Operario D, Zhao Y, Shi A, Zhang Z, et al. Effects of a mobile health intervention to promote HIV selftesting with MSM in China: a randomized controlled trial. AIDS Behav. 2019;23(11):3129-39.

27. Ybarra ML, et al. Pilot RCT results of an mHealth HIV prevention program for sexual minority male adolescents. Pediatrics. 2017;140(1):e20162999.

28. Stephenson R, et al. Project Moxie: results of a feasibility study of a telehealth intervention to increase HIV testing among binary and nonbinary transgender youth. AIDS Behav. 2019;24(5):15171530. This pilot RCT of an online video counseling session for HIVST is unique for its tailoring to transgender and nonbinary youth and because it is a brief intervention approach.

29. Horvath KJ, et al. The feasibility, acceptability and preliminary impact of mobile application to increase repeat HIV testing among sexual minority men. AIDS Behav. 2019;24(6):1835-1850.

30. Bauermeister JA, Tingler RC, Demers M, Connochie D, Gillard G, Shaver J, et al. Acceptability and preliminary efficacy of an online HIV prevention intervention for single young men who have sex with men seeking partners online: the myDEx Project. AIDS Behav. 2019;23(11):3064-77.

31. Thomas B, Closson EF, Biello K, Menon S, Navakodi P, Dhanalakshmi A, et al. Development and open pilot trial of an HIV-prevention intervention integrating mobile-phone technology for male sex workers in Chennai, India. Arch Sex Behav. 2017;46(4):1035-46.

32. Sullivan PS, Driggers R, Stekler JD, Siegler A, Goldenberg T, McDougal SJ, et al. Usability and acceptability of a mobile comprehensive HIV prevention app for men who have sex with men: a pilot study. JMIR Mhealth Uhealth. 2017;5(3):e26 This singlearm pilot trial of an HIV testing intervention for MSM is an example of a comprehensive HIV and sexual health mobile application that provides information and access to a wide variety of prevention strategies (e.g., PrEP information) and resources (e.g., condoms).

33. McCoy SI, et al. Stick To It: pilot study results of an intervention using gamification to increase HIV screening among young men who have sex with men in California. Mhealth. 2018;4:40.

34. De Boni RB, et al. An internet-based HIV self-testing program to increase HIV testing uptake among men who have sex with men in Brazil: descriptive cross-sectional analysis. J Med Internet Res. 2019;21(8):e14145 This cross-sectional study is a large-scale implementation of an online site in Brazil that provides relevant information and the ability to order HIVST kits.

35. Janssen R, et al. Alone but supported: a qualitative study of an HIV self-testing app in an observational cohort study in South Africa. AIDS Behav. 2020;24(2):467-74.
36. Njuguna $\mathrm{N}$, et al. The effect of human immunodeficiency virus prevention and reproductive health text messages on human immunodeficiency virus testing among young women in rural Kenya: a pilot study. Sex Transm Dis. 2016;43(6):353-9 This quaziexperimental study of a text messaging HIV testing intervention is unique for being tailored for women in Sub-Saharan Africa.

37. Horvath KJ, et al. Using technology to assess and intervene with illicit drug-using persons at risk for HIV. Curr Opin HIV AIDS. 2017;12(5):458-66.

38. Muessig KE, et al. Recent mobile health interventions to support medication adherence among HIV-positive MSM. Curr Opin HIV AIDS. 2017;12(5):432-41.

39. Iott $\mathrm{BE}$, et al. Comparative analysis of recruitment strategies in a study of men who have sex with men (MSM) in Metropolitan Detroit. AIDS Behav. 2018;22(7):2296-311.

40. Freeman AE, et al. Perceptions of HIV self-testing among men who have sex with men in the United States: a qualitative analysis. AIDS Educ Prev. 2018;30(1):47-62.

41. $\mathrm{Xu} \mathrm{W,} \mathrm{Zheng} \mathrm{Y,} \mathrm{Kaufman} \mathrm{MR.} \mathrm{Predictors} \mathrm{of} \mathrm{recent} \mathrm{HIV} \mathrm{testing}$ among Chinese men who have sex with men: a barrier perspective. AIDS Patient Care STDS. 2018;32(10):408-17.

42. Bauermeister JA, et al. Acceptability and preliminary efficacy of a tailored online HIV/STI testing intervention for young men who have sex with men: the Get Connected! program. AIDS Behav. 2015;19(10):1860-74.

43. GMSA. The mobile economy: Sub-Saharan Africa 2019. [cited 2020 April 24]; Available from: https://www.gsma.com/ mobileeconomy/wp-content/uploads/2020/03/GSMA MobileEconomy2020_SSA_Eng.pdf.

44. Pew Research Center. Mobile fact sheet. [cited 202 April 24]; Available from: https://www.pewresearch.org/internet/fact-sheet/ mobile/

45. Almirall D, et al. Introduction to SMART designs for the development of adaptive interventions: with application to weight loss research. Transl Behav Med. 2014;4(3):260-74.

46. Sprague C, et al. Towards ending the US HIV epidemic by 2030: understanding social determinants of health and HIV in Mississippi. Glob Public Health. 2020;15(1):31-51.

47. Mee P, et al. The development of a localised HIV epidemic and the associated excess mortality burden in a rural area of South Africa. Glob Health Epidemiol Genom. 2016;1:e7.

48. Hightow-Weidman L, Muessig K, Claude K, et al. Maximizing digital interventions for youth in the midst of COVID-19: lessons from the Adolescent Trials Network for HIV Interventions. AIDS Behav. 2020. https://doi.org/10.1007/s10461-020-02870-w.

49. Carrico AW, Horvath KJ, Grov C, et al. Double jeopardy: methamphetamine use and HIV as risk factors for COVID-19. AIDS Behav. 2020. https://doi.org/10.1007/s10461-020-02854-w.

Publisher's Note Springer Nature remains neutral with regard to jurisdictional claims in published maps and institutional affiliations. 